\title{
Boundary Layer Impact on Mountain Waves across Western Ghats of India
}

\author{
Naresh Kumar, ${ }^{*}$ M. Mohapatra* and B. P. Yadav*
}

\begin{abstract}
A two- layer model has been developed assuming a steady, non- rotating and frictionless flow of vertically unbounded Boussinesq fluid across 2-D profile of Western Ghats of India. Further, it is assumed that lower layer is inviscid with neutral stability $(\mathrm{N}=0)$ and upper layer has constant static stability with respect to height, given by $N=$ Constant (where $N$ is Brunt- Väisälä frequency). The analytical expressions for vertical velocities and mountain drag have been derived across Western Ghats of India. It is found that vertical velocities are dependent on the characteristics like size and height of ridge as well as plateau region to the eastern side of the Western Ghats. However, the mountain drag is not dependent on the above characteristics of the plateau region to the east of Western Ghats confirming the earlier findings.
\end{abstract}

Keywords: Mountain drag, vertical velocity and Western Ghats

\section{Introduction}

Orographic barriers play a very important role in distribution of rainfall over a region. In India, the location and orientation of major hill ranges including Western Ghats, Eastern Ghats, Khasi- Jayantia hills, Assam- Burma hills and Pirpanjal hills of Kashmir valley play

*India Meteorological Department, New Delhi 110003; naresh.nhac@gmail.com 
major role in occurrence and intensity distribution of rainfall. There are many studies on the role of these orographic barriers. Dutta (2001) has proposed a two dimensional model to study the momentum flux and Energy flux across the Western Ghats of India considering Brunt-Väisälä frequency as constant throughout the atmosphere. His studies show that momentum flux and Energy flux are independent of the plateau parts of Ghats. Dutta and Naresh (2005) have done studies related to mountain drag, momentum flux and Energy flux associated with mountain waves across Assam- Burma hills of India by using the Radiosonde data of Guwahati. Their study also shows the role of valley between the ridges of hills on drag and fluxes. Naresh et al. (2005) have evaluated the role of earth rotation on mountain drag across KhasiJayantia hills of India. Recently, Naresh and Hatwar (2008) have considered a two-dimensional rotating flow associated with mountain wave for wind with shear. They obtained the expression for mountain drag across a ridge using asymptotic technique. All the above studies are related to single layer models considering Brunt-Väisälä frequency as constant throughout the atmosphere. These studies are limited as the frequency does not remain constant and varies with respect to height. Considering the above, a study has been undertaken to develop a two layer model with a lower layer characterized by neutral stability (Brunt-Väisälä frequency $=0$ ) and upper layer characterized by constant stability (Brunt-Väisälä frequency= A constant), to calculate the vertical velocity and mountain drag in association with the orographic waves across Western Ghats of India. The model development has been discussed in section 2 . The results and discussions are presented in section 3 and the broad conclusions are presented in section 4 .

\section{Two Layer Model for Vertical Velocities and Mountain Drag}

The methodology to derive analytical expression for vertical velocities across the Western Ghats is presented in section 2.1. The methodology to derive the analytical expression for mountain drag across Western Ghats is presented in section 2.2. 


\subsection{Vertical velocities across Western Ghats of India}

A two- dimensional stably stratified flow over mountain ridges assuming the basic flow wind and basic state density as constant has been considered. Following Dutta and Naresh (2005) for steady state, linear, non- rotating, frictionless and Boussinesq flow reduces to

$$
\frac{\partial^{2} \hat{w}}{\partial z^{2}}+\left(\frac{N^{2}}{U^{2}}-k^{2}\right) \hat{w}=0
$$

where, $\mathrm{N}$ is Brunt- Väisälä frequency; $\mathrm{U}$ basic flow wind. $\hat{w}$ is the Fourier transform of the perturbation vertical velocity $w^{\prime}$.

In lower layer, it is assumed $0<z<D, N=0$ and $U=$ Cons $\tan t$.

The equation (1) has been solved using following lower boundary condition:

$$
w^{\prime}(x, z=0)=U \frac{\partial h}{\partial x}
$$

where, $h(x)$ is the 2-D profile of Western Ghats and is defined by

$$
h(x)=\frac{H}{1+\frac{x^{2}}{a^{2}}}+b \tan ^{-1} \frac{x}{a}, \text { Sarker (1965) }
$$

where, $a=18.0 \mathrm{~km}, H=.52 \mathrm{~km}, b=\frac{2}{\pi} \times .35 \mathrm{~km}$.

Following Broad (2002), the solution of the equation (1) in lower layer by using Fourier transforms of the equation (2) may be written as:

$$
\hat{w}(k, z)=i k U \hat{h}(k) \exp (-k z)
$$

where, $\hat{h}(k)$ is the Fourier transform of $h(x)$ and $\mathrm{k}$ is the wave number. 
The inverse Fourier transform of the equation (4) gives

$$
w^{\prime}(x, z)=i U \int_{-\infty}^{\infty} k \hat{h}(k) e^{-(z-i x) k} d k
$$

Substituting Fourier transform of the equation (3) into the equations (5), we have

$$
w^{\prime}(x, z)=\frac{-2 H U a x(a+z)}{\left((a+z)^{2}+x^{2}\right)^{2}}+b U \frac{(a+z)}{(a+z)^{2}+x^{2}}
$$

The equation (6) is the expression for perturbation vertical velocity in lower layer.

In upper layer, it is assumed that $z \geq D, N=$ Cons $\tan t$ and $U=$ Cons $\tan t$.

The equation (1) under hydrostatic assumption reduces to $\frac{\partial^{2} \hat{w}}{\partial z^{2}}+l^{2} \hat{w}=0$, where, $l=\frac{N}{U}$ is the Scorer's parameter.

The solution of the above equation using the equation (4) as a lower boundary condition at $z=D$ and allowing the Energy to propagate at great height becomes

$$
\hat{w}(k, z)=i k U \hat{h}(k) \exp (i l(z-D)) \exp (-D k)
$$

By its inverse Fourier transform

$$
w^{\prime}(x, z)=i U \exp (i l(z-D)) \int_{-\infty}^{\infty} k \hat{h}(k) e^{-(D-i x) k} d k
$$

Substituting the Fourier transform of equation (3) into the equations (8), we have

$$
\begin{aligned}
w^{\prime}(x, z)= & \frac{-H U a}{\left((a+D)^{2}+x^{2}\right)^{2}}\left[\begin{array}{l}
2(a+D) x \cos l(z-D) \\
+\left\{(a+D)^{2}-x^{2}\right\} \sin l(z-D)
\end{array}\right] \\
& +\frac{b U}{(a+D)^{2}+x^{2}}[(a+D) \cos l(z-D)-x \sin l(z-D)]
\end{aligned}
$$


The equation (9) is the expression for hydrostatic perturbation vertical velocity in upper layer. First part in the equation (9) is vertical velocity due to ridge of Western Ghats and second part is due to its plateau part.

\subsection{Mountain Drag}

Following Dutta and Naresh (2005), the expression for mountain drag ' $\mathrm{F}$ ' may be written as

$$
F=-2 \pi \rho_{0} i \int_{0}^{\infty} \frac{1}{k} \hat{w} * \frac{\partial \hat{w}}{\partial z} d k
$$

where, $\hat{w}^{*}$ is the complex conjugate of $\hat{w}$ and $\rho_{0}$ is basic state density.

Using the equations (7) into the equation (10), we have

$$
F=2 \pi N \rho_{0} U \int_{0}^{\infty} k \hat{h}(k) \hat{h}^{*}(k) e^{-2 D k} d k
$$

Finally, substituting the Fourier transform of the equation (3) into the equation (11) gives

$$
F=\frac{1}{2} \pi N \rho_{0} U H^{2} \frac{1}{\left(1+\frac{D}{a}\right)^{2}}
$$

The equation (12) is the mountain drag across Western Ghats, which depend upon the half width as well as depth of the lower layer of the mountain, whereas, it is not true in case of single layer model as shown by Dutta (2001). 


\section{Result and discussions}

The analytical expressions for vertical velocities and mountain drag across WG have been derived. From the equations (6) and (9), it may be concluded that vertical velocities for WG are no longer independent on the plateau part of the Ghats. The contours of vertical velocities for $U=10 \mathrm{~ms}^{-1}$ and $D=1 \mathrm{~km}$ are shown in the figures (1) and (2) respectively.

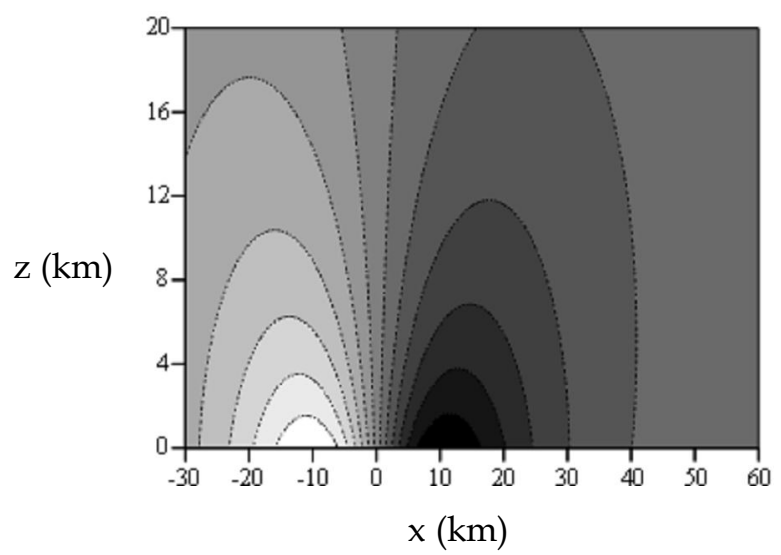

Figure 1: Vertical velocity contours for lower layer

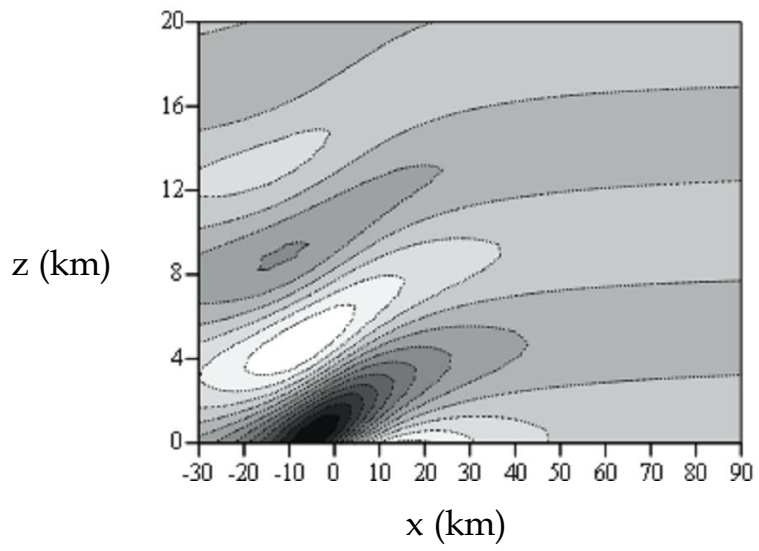

Figure 2: Vertical velocity contours for upper layer

The equation (12) shows that mountain drag no longer independent on the half width of the WG, but in case of single layer model, it is not true. This may be due to fact that mountain drag at surface for single layer model depends upon the surface wind, height of the mountain and brunt 
Väisälä frequency, but for upper layer model, the shape of mountain also plays an important role. By substituting $D=0$ into the equation (12),

$$
F_{W G}=\frac{1}{2} \pi N U H^{2}
$$

The equation (13) is the same expression of mountain drag for WG as obtained by Dutta (2001).

\section{Conclusion}

1. The analytical expressions for vertical velocities and mountain drag have been derived across Western Ghats of India.

2. It is found that vertical velocities are dependent on the characteristics like size and height of ridge as well as plateau region to the eastern side of the Western Ghats.

3. Mountain drag is not dependent on the above characteristics of the plateau region to the east of Western Ghats confirming with Dutta (2001).

\section{Reference}

[1] Broad, A.S. (2002). The impact of the atmospheric boundary layer on mountain forced gravity waves. Proceedings, 10th Conference on Mountain Meteorology and MAP Meeting 2002.

[2] Dutta, S.N. (2001). Momentum flux, Energy flux and pressure drag associated with mountain wave across Western Ghats. Mausam, 52 (2): 325-332.

[3] Dutta, S.N. and Naresh Kumar (2005). Parameterization of momentum and Energy flux associated with mountain wave across Assam-Burma hills. Mausam, 56 (3), 527-534.

[4] Naresh Kumar and Hatwar, H. R. (2008). A Study of mountain drag for wind with shear in a stratified rotating flow, International Journal of Fluid Mech. Res. 35, 1, 51-59.

[5] Naresh Kumar, Roy Bhowmik, S. K., Ahmed, N. and Hatwar, H. R. (2005). A Mathematical Model for Rotating Stratified Airflow across Khasi-Jayantia hills, Mausam, 56, 4, 771-779.

[6] Sarker, R.P. (1965). A theoretical study of mountain waves on Western Ghats, Indian journal of meteorology and geophysics, 16 (4), 563-584. 